Service social

\title{
Les modèles de rattachement du service social en milieu hospitalier. Des faits, des enjeux et des défis
}

\section{Georgette Béliveau et Lise Hébert}

Volume 45, numéro 3, 1996

Santé

URI : https://id.erudit.org/iderudit/706735ar

DOI : https://doi.org/10.7202/706735ar

Aller au sommaire du numéro

Éditeur(s)

École de service social de l'Université Laval

ISSN

1708-1734 (numérique)

Découvrir la revue

Citer cet article

Béliveau, G. \& Hébert, L. (1996). Les modèles de rattachement du service social en milieu hospitalier. Des faits, des enjeux et des défis. Service social, 45(3), 9-30. https://doi.org/10.7202/706735ar
Résumé de l'article

Cet article vise à faire le point sur la question des modèles de rattachement administratif du service social en centre hospitalier et sur les changements récents d'orientation dans ce domaine. Le point de départ est une brève perspective historique du service social dans ce milieu de pratique, principalement au Québec. On y présente les différents modèles de rattachement d'un service social aux centres hospitaliers. Par la suite, on élabore un modèle contemporain qui semble s'imposer de plus en plus - la structure par programmes -, en faisant ressortir les ressemblances et les différences de ce dernier avec ce qui a déjà existé au Québec, ce qui permet de discuter des avantages et des inconvénients de chaque modèle. L'article se termine par une réflexion sur les différents enjeux et sur les possibilités qui s'offrent au service social dans la conjoncture actuelle, les auteures faisant ressortir les défis pour l'avenir du service social, dans un tel contexte de pratique. 


\section{A $R$ R $T$ I $/ C$ L E S}

\section{Les modèles de rattachement du service social en milieu hospitalier Des faits, des enjeux et des défis}

Georgette BÉLIVEAU Travailleuse sociale et professeure titulaire Université Laval

Lise HÉBERT Travailleuse sociale au Centre hospitalier universitaire de Québec Étudiante au doctorat en service social

Nous visons, par le présent article, à faire le point sur la question des modèles de rattachement administratif du service social en centre hospitalier et sur les changements récents d'orientation dans ce domaine. L'occasion est bonne, aussi, de réfléchir sur les enjeux et sur les possibilités qui s'offrent au service social dans la conjoncture actuelle.

L'article présente les différents modèles de rattachement d'un service social en milieu hospitalier. Son point de départ est une brève perspective historique du service social dans ce milieu de pratique, principalement au Québec. Cette vision nous permettra, par la suite, d'élaborer un modèle contemporain qui 
semble s'imposer de plus en plus dans les centres hospitaliers la structure par programmes -, de voir quelles sont les ressemblances et les différences de ce modèle avec ce qui a déjà existé au Québec et de discuter ensuite de ses avantages et de ses inconvénients. Nous terminerons cet article en faisant ressortir les enjeux et les défis pour l'avenir du service social en milieu hospitalier, dans un tel contexte de pratique ${ }^{1}$.

\section{LE DÉVELOPPEMENT DU SERVICE SOCIAL EN MILIEU HOSPITALIER}

Elizabeth Taylor Rossinger (1985), dans son «Tableau synoptique sur le développement du service social en milieu de santé », fait une rétrospective du service social en milieu de santé au Québec en replaçant les événements dans un contexte nord-américain et européen. Elle situe le début du service social en milieu hospitalier au XIX ${ }^{\mathrm{e}}$ siècle, principalement en Angleterre et aux ÉtatsUnis.

En 1875, Sir Charles Loch, secrétaire du Charity Organization Society (C.O.S.) de Londres ${ }^{2}$, s'employait, en collaboration avec des médecins, à diriger des patients vers les C.O.S. et à offrir à ces personnes un service d'investigation sociale. Il faisait observer que les hôpitaux fonctionnaient selon les besoins des médecins, plutôt que selon ceux de la communauté (Macman, 1990, p. 7-22). Dix ans plus tard, les C.O.S. introduisaient des programmes de service social dans sept hôpitaux.

En 1905, le Dr Richard Cabot, chef du département de médecine du Massachusetts General Hospital (Boston), était convaincu de l'influence des problèmes psychosociaux sur la maladie et croyait à l'apport complémentaire que le «Social Service Program» pourrait offrir dans le milieu (Sabetta, 1981, p. 7-18). En 1914, on engage à cet hôpital la première chef du service social. C'est aussi au début du $\mathrm{XX}^{\mathrm{e}}$ siècle que le service social fait son apparition au Québec, avec la fondation de l'École de McGill en 1918. Il s'implante alors surtout dans les milieux anglophones, comme à l'hôpital Royal Victoria où l'on forme un département de service social en 1919. Le service social prend aussi place dans les hôpitaux psychiatriques de Montréal. À la même époque, il se développe en Europe et prend de l'importance aux États-Unis. Il faut attendre les années 1920 et 1930 pour voir le service social s'implanter dans les milieux francophones 
québécois. Après la Seconde Guerre mondiale, le service social hospitalier élargit son champ d'action, précise ses rôles et se répand davantage au Québec: on parle désormais de service social en milieu de santé. L'Institut de réadaptation de Montréal puis l'Hôpital Saint-Jean-de-Dieu et l'Hôpital MaisonneuveRosemont sont parmi les premiers à engager des travailleurs sociaux en milieu hospitalier francophone.

Dans la région de Québec, c'est davantage au cours des années 1950 que l'on associe le service social au milieu hospitalier (St-Arnaud, 1997, p. 3-29). Dans les années 1960, on insiste beaucoup sur les services en milieu psychiatrique et on trouve de plus en plus de publications sur le sujet. Entre autres, Harriett M. Bartlett (1961) propose son mode d'analyse d'un champ de pratique : étude des éléments essentiels de la pratique du service social, caractéristiques du champ de pratique et caractéristiques de la pratique du service social dans un milieu donné. Ce modèle est alors enseigné et souvent appliqué au milieu hospitalier.

\section{LES MODÈLES DE RATTACHEMENT ADMINISTRATIFS ET LES CARACTÉRISTIQUES ORGANISATIONNELLES DU SERVICE SOCIAL EN MILIEU HOSPITALIER}

Très peu d'auteurs, cependant, se sont penchés sur les caractéristiques organisationnelles du service social en milieu hospitalier et ont dégagé les conséquences de cette organisation sur la pratique même du service social dans ces différents contextes. Nous reprenons et analysons donc dans le présent article les principaux modèles organisationnels du service social en milieu hospitalier au Québec depuis les années 1960 ; nous y étudions ensuite, dans un dernier volet et à la lumière de la même grille d'analyse, un modèle prôné par les gestionnaires d'hôpitaux de cette fin de siècle. Les lecteurs pourront, à leur guise, adapter ces propos à la réalité du modèle d'organisation existant ou en émergence dans leur milieu et surtout voir comment tirer parti d'une situation qu'ils n'ont pas toujours désirée.

Depuis les années 1960 environ, moment où le service social s'est vraiment imposé dans plusieurs hôpitaux du Québec, plusieurs modèles de rattachement ont parfois coexisté. Ainsi le service social: 
- a eu des travailleurs sociaux rattachés aux divers chefs de départements cliniques d'un centre hospitalier ;

- a été rattaché à un centre hospitalier ayant un département de service social et un chef de service travailleur social;

- a été rattaché à une direction spécialisée d'un centre de services sociaux;

- a été rattaché à une filiale d'un centre de services sociaux.

Voyons maintenant les caractéristiques de chacune des formes d'organisation ${ }^{3}$, les objectifs poursuivis dans chacun des contextes, les formes de travail d'équipe, les caractéristiques du travail professionnel, les modèles d'identification professionnelle et les qualités requises.

\section{Les travailleurs sociaux sont rattachés aux divers chefs de départements cliniques d'un centre hospitalier}

Ce modèle d'organisation du service social a été et est très peu répandu au Québec. Cependant, on peut le confondre facilement avec le mode d'organisation mis en évidence actuellement dans plusieurs centres hospitaliers où l'on propose de rattacher les travailleurs sociaux aux divers programmes. Ce dernier modèle, emprunté au vocabulaire des écoles d'administration, fera l'objet d'une attention spéciale dans une partie subséquente de cet article.

Lorsqu'un modèle de rattachement aux divers chefs de départements cliniques est adopté, les travailleurs sociaux sont payés par le centre hospitalier à même le budget du département auquel ils appartiennent. Il n'existe habituellement aucun contrat de service et la nature des services dépend fréquemment de la demande du chef de service clinique, qui est le plus souvent un médecin. Ce sont les objectifs du département qui priment, suivis des objectifs du centre hospitalier. Les objectifs sociaux demeurent éloignés et doivent être régulièrement et fermement défendus.

Le travail d'équipe, qu'il soit formel ou informel, est principalement influencé par le modèle médical. Il est alors difficile de faire en sorte que les valeurs sociales influencent la gouverne de l'hôpital.

Dans un tel contexte, le travailleur social ne trouve sur les lieux à peu près aucun soutien professionnel propre à sa discipline. 
Ce soutien ne peut venir que d'un regroupement professionnel extérieur au département et le plus souvent extérieur au centre hospitalier. Dans ces milieux, l'intervenant, surtout s'il est nouveau et peu expérimenté, peut avoir tendance à s'identifier uniquement aux valeurs médicales et aux sciences de la santé. Il peut s'ensuivre une perte d'identification professionnelle au service social.

Les travailleurs sociaux qui exercent dans de tels milieux doivent posséder une expérience antérieure solide, et il est souvent préférable qu'ils détiennent une maîtrise en service social. Le critère de sélection le plus important est cependant l'identification à la profession.

\section{Le service social est rattaché à un centre hospitalier avec un département de service social et un chef de service travailleur social}

Ce modèle de rattachement où le service social est la responsabilité du centre hospitalier qui en supporte les coûts fut très répandu lorsque le service social s'est implanté dans les hôpitaux au Québec. Il a pour caractéristique de comporter un département de service social autonome géré par un professionnel du service social. Ce modèle a persisté jusqu'à ce que les centres de services sociaux soient mis sur pied. Il est réapparu, dans beaucoup de centres hospitaliers (CH), en 1993, avec la réorganisation des établissements du réseau.

Lorsqu'un département de service social est formé dans un centre hospitalier de structure hiérarchique avec état-major, le département de service social peut être qualifié d'état-majorservice $^{4}$ et son chef joue un rôle de conseil à l'égard des départements relevant de la structure hiérarchique, c.-à-d. ceux qui sont en lien direct avec la mission de l'établissement, comme les soins aux patients. Les gestionnaires ou cadres hiérarchiques voués à la réalisation de la mission du centre hospitalier sont aidés dans leur travail par des experts de différents domaines, dont le chef du service social.

Le département de service social peut aussi faire partie d'une structure fonctionnelle du centre hospitalier, et ce fut habituellement le cas jusqu'à présent. Le service social, qui est alors un regroupement basé sur la fonction qu'il assume dans l'organisation, vient soutenir la mission première de l'hôpital. 
En ce sens, il joue un rôle secondaire mais complémentaire et important. On confie alors la responsabilité du département de service social à un chef de service travailleur social qui a un pourvoir hiérarchique sur son personnel. Le chef de service, en plus d'être responsable de la qualité des actes professionnels, est chargé du contrôle des procédés ${ }^{5}$, des politiques et des pratiques particulières. Le service social relève alors d'une direction fonctionnelle ${ }^{6}$, du centre hospitalier habituellement, soit la direction des services hospitaliers, soit la direction des services professionnels.

La nature des services sociaux dépend de l'entente intervenue entre le chef du service social et la direction du centre hospitalier. Dans un tel cas, il y a habituellement prédominance des objectifs du centre hospitalier et des objectifs de santé, alors que les objectifs sociaux sont parfois mis en sourdine ou doivent être régulièrement et fermement défendus. Les travailleurs sociaux sont appelés à participer à différentes formes d'équipes dans lesquelles le modèle médical est prédominant. Certaines équipes sont rattachées au travail clinique, alors que d'autres se présentent sous forme de participation aux équipes mises sur pied par le centre hospitalier lui-même ou sont reliées à la gestion de l'établissement. Il est important pour le travailleur social d'accepter toute forme de participation à tous les niveaux de l'hôpital et même d'offrir ses services pour s'assurer du respect et de la promotion des valeurs et du rôle spécifique du service social dans le milieu.

Le modèle médical dominant dans un tel contexte influence la pratique professionnelle en service social et l'on insiste pour que les travailleurs sociaux centrent leurs interventions sur les faits et agissent avec rapidité. Le travail peut être facilité s'il y a une équipe de travailleurs sociaux suffisamment nombreuse pour que les intervenants se soutiennent mutuellement ou, dans le cas de petites unités, si les intervenants sociaux peuvent compter sur l'appui d'un regroupement régional ou provincial de travailleurs sociaux intéressés à la santé. Le travail demande alors une grande autonomie professionnelle et une excellente identification à la profession. Les valeurs médicales prédominantes dans le milieu peuvent rendre plus difficile cette identification au service social. Pour assurer l'efficacité et le professionnalisme du service et pour maintenir l'identification au service social, on devrait embaucher pour de tels postes des bacheliers en service social avec de bonnes expériences de travail ou des titulaires d'une maîtrise. Le travail peut se révéler très stimulant 
pour les professionnels du service social qui aiment les défis, surtout s'il se trouve une bonne équipe sur place. Dans un contexte favorable, un stagiaire en service social bien encadré peut profiter de son expérience.

\section{Le service social est rattaché à une direction spécialisée d'un centre de services sociaux}

Le mode d'organisation où le service social est rattaché à une direction spécialisée d'un centre de services sociaux (CSS) a dominé des années 1970 à la dernière réforme des services sociaux, au début des années 1990. Il a donné lieu à des expériences de stage intéressantes et a permis d'accueillir de jeunes professionnels.

Dans ce modèle, les travailleurs sociaux sont engagés et payés par un centre de services sociaux (CSS) et prêtés au centre hospitalier par un contrat de service. Au CSS, les travailleurs sociaux en milieu hospitalier sont la plupart du temps regroupés sous une même direction ou selon un programme clientèle. On a alors une départementalisation en fonction du canal de distribution ou par clientèle ${ }^{7}$.

En ce qui a trait au département de service social à l'intérieur du centre hospitalier, sa structure et l'autorité de chef de service sont les mêmes que dans le modèle du service social rattaché à un centre hospitalier avec un département de service social et un chef de service qui est travailleur social. Il s'agit du même modèle de structure organisationnelle quant à l'insertion du service social dans le $\mathrm{CH}$. Par ailleurs, si l'on considère le service social comme entité à l'intérieur du centre de services sociaux, on constate qu'il participe directement à la mission du CSS, entrant directement dans la ligne hiérarchique de production de services à l'intérieur d'une organisation de type hiérarchique qui elle-même a son état-major (Bergeron, 1989).

Toutefois, la double appartenance des intervenants fait en sorte que ces derniers sont partagés entre les objectifs et les façons de faire du centre de services sociaux et ceux du centre hospitalier. Il s'agit d'une situation qui a fait l'objet de critiques en ce qui a trait à la complexité et à la lourdeur administrative (Émond, 1985).

En milieu hospitalier, il existe différentes formes d'équipes: les unes sont dites formelles et les autres informelles. Certaines équipes de travail sont prévues dans le contrat de service. 
Le travail professionnel adopte alors un modèle social qui tient compte du milieu médical et de ses valeurs. L'appartenance au centre de services sociaux favorise le soutien de collègues engagés dans des milieux semblables. La double allégeance permet un programme de service social qui tient compte à la fois des volets santé et services sociaux et fait le lien entre santé et problèmes sociaux. Un tel contexte offre la possibilité d'acquérir une très forte identité professionnelle grâce au soutien des collègues travailleurs sociaux du milieu de la santé. On peut trouver des intervenants de tous les niveaux de formation; qu'ils soient conseillers sociaux avec expérience, bacheliers en service social ou diplômés de la maîtrise, ils peuvent rendre des services.

\section{Le service social est rattaché à une filiale d'un centre de services sociaux}

Ce modèle de rattachement, comme celui qui précède, fut très répandu des années 1970 à la dernière réforme des services sociaux au début des années 1990.

Dans ce modèle, les travailleurs sociaux sont engagés et payés par un centre de services sociaux (CSS) et prêtés au centre hospitalier par un contrat de service. Tout ce qui a été dit au sujet du service social rattaché à une direction spécialisée d'un centre de services sociaux est également vrai ici. Cependant, au centre de services sociaux, les travailleurs sociaux en milieu hospitalier sont tous regroupés avec des travailleurs sociaux intéressés par différentes problématiques sur la base d'un même territoire: on a alors une départementalisation selon le territoire. Cette structure, et l'accent qui est mis sur le territoire desservi, fait en sorte que les objectifs du centre de services sociaux et les objectifs sociaux dominent et qu'il peut y avoir des problèmes d'harmonisation avec les objectifs du centre hospitalier.

Au point de vue du travail d'équipe, la collaboration et les échanges s'établissent d'abord avec les travailleurs sociaux de la filiale du centre de services sociaux, puis avec les autres professionnels du milieu de la santé. Il existe parfois différentes équipes formelles et informelles.

Le travail professionnel adopte alors un modèle social. Le centre hospitalier est un point de service où l'on rend des services sociaux, que les problèmes aient ou non un lien avec la santé. On rend habituellement surtout des services concrets. Un tel contexte offre la possibilité de développer une très forte 
identité professionnelle grâce au soutien des collègues. La direction du centre de services sociaux a tendance à employer des conseillers sociaux avec expérience pour faire ce travail.

\section{LE MODÈLE CONTEMPORAIN : LES TRAVAILLEURS SOCIAUX SONT RATTACHÉS AUX DIVERS PROGRAMMES D'UN CENTRE HOSPITALIER}

Ce modèle d'organisation, basé sur la notion de programme, semble gagner de la popularité auprès des administrateurs d'hôpitaux, surtout dans les milieux universitaires. On en retrouve diverses variantes: nous en verrons deux, l'une de type matriciel et l'autre de type intégrateur. Dans les deux cas, cependant, les programmes peuvent être formulés selon différentes logiques: regroupement de services cliniques, niveaux de soins à prodiguer, santé publique, types de clientèle, caractère interne ou externe des services, etc. (Collège canadien des directeurs des services de santé, 1994). C'est un modèle qui prend ses origines aux États-Unis dans les années 1950 pour la forme matricielle et dans les années 1970 pour la forme intégratrice. Au cours des dix dernières années le modèle a évolué et s'est développé dans les hôpitaux canadiens de l'Ouest et de l'Ontario, tels Sunnybrook Health Sciences Center (Toronto) et University of Alberta Hospital (Association des hôpitaux du Québec, 1996).

Le modèle matriciel ${ }^{8}$ est une façon de donner des services professionnels dans différents programmes, tout en maintenant le regroupement des travailleurs sociaux ${ }^{9}$. Le modèle combine les activités des différentes fonctions d'une organisation et est étroitement lié à la gestion de projet. Le personnel et le matériel des unités fonctionnelles sont "prêtés", de façon temporaire ou non, à une unité donnée pour réaliser un programme, une activité ou un projet. Ce modèle se situe entre la départementalisation fonctionnelle et la départementalisation selon le projet ou le produit. Le travailleur social est alors intégré à la fois à un programme du centre hospitalier et à une équipe de travailleurs sociaux. Le budget demeure la responsabilité du service social en tant que service. Avec cette structure, le travailleur social maintient une double appartenance: son action est liée au développement des activités spécifiques du programme, de même qu'au développement du service social comme entité dans le centre hospitalier. 
Dans le milieu hospitalier, on appelle Patient-Focused Care une forme plus intégratrice des divers professionnels dans chaque programme. Pour reprendre les termes de Pierre-G. Bergeron, on a alors une départementalisation par "produits» ou par programmes-clientèles. Le travailleur social relève exclusivement des autorités du programme auquel il est intégré. Un seul cadre détient l'autorité hiérarchique et celle-ci peut être assumée par un professionnel d'une autre discipline que le service social. Sur le plan administratif, le travailleur social est intégré à l'équipe multidisciplinaire et relève du même cadre hiérarchique que les autres membres de cette équipe multidisciplinaire. La structure par programmes dite Patient-Focused Care est la forme la plus populaire actuellement (Association des hopitaux du Québec, 1996). Dans ce modèle, il y a prédominance des objectifs du programme et ensuite des objectifs du centre hospitalier. Les objectifs sociaux demeurent, mais ils sont liés aux objectifs du programme.

Dans les deux variantes que nous venons de décrire, les travailleurs sociaux ont ou peuvent se donner l'occasion de participer à plusieurs types d'équipes. La forme la plus connue est l'équipe clinique issue du modèle médical et existant dans tous les modèles d'organisation des services sociaux en milieu hospitalier. Étant donné l'importance de l'idéologie du modèle administratif, le travailleur social se doit d'être à l'affût de ce qui se passe dans le centre hospitalier. Il saisira toutes les possibilités de participer à des équipes de gestion. Il lui faudra se rappeler que les différents professionnels, membres des équipes multidisciplinaires de type clinique ou administratif, peuvent être en compétition les uns avec les autres.

Dans le modèle Patient-Focused Care, le soutien professionnel existe peu ou pas sur les lieux à moins que les travailleurs sociaux n'en mettent eux-mêmes sur pied. Ce soutien professionnel peut venir d'un regroupement de travailleurs sociaux à l'intérieur du milieu hospitalier, d'un regroupement de travailleurs sociaux en santé ou d'un regroupement professionnel plus large.

Qu'il s'agisse du modèle matriciel, et encore plus s'il s'agit du modèle Patient-Focused Care, les professionnels qui décident de travailler dans ces milieux doivent miser sur d'excellentes qualifications professionnelles au niveau du baccalauréat, de la maîtrise et même du doctorat, sur de bonnes expériences antérieures et faire preuve d'une personnalité forte et très bien identifiée à la profession. Ils doivent posséder une vision large du 
service social, une grande autonomie. Il faut se rappeler que la qualité du travail professionnel repose uniquement sur les intervenants sociaux, qui sont les meilleurs ambassadeurs de la pertinence du travail social. Les travailleurs sociaux doivent individuellement promouvoir les valeurs du service social. Certains travailleurs sociaux, insuffisamment avertis ou formés, risqueraient de s'identifier presque uniquement aux valeurs médicales et aux sciences de la santé. Il peut s'ensuivre une perte d'identification professionnelle au service social et une absence de valorisation de cette dernière.

\section{Les avantages et les inconvénients des principaux modèles de structures organisationnelles}

Nous présentons ici les avantages et les inconvénients des principaux modèles de structures organisationnelles déjà expliquées, utilisés dans le passé et encore maintenant dans les centres hospitaliers. Nous nous sommes inspirées de l'ouvrage déjà cité de Pierre-G. Bergeron pour définir les avantages et les inconvénients liés à ces modèles.

La structure hiérarchique doublée d'un état-major est présente, comme nous l'avons expliqué, et ce, à des degrés divers, dans le modèle rattachant le service social à un centre hospitalier avec un département de service social et un chef de service, travailleur social. Elle l'est aussi dans le modèle de service social rattaché à une direction spécialisée d'un centre de services sociaux et dans celui du service social rattaché à une filiale d'un centre de services sociaux. Cette structure dite hiérarchique présente l'avantage de la spécialisation, en ce sens que chacun apporte l'expertise professionnelle de sa propre discipline. Cette expertise se transmet horizontalement aux pairs et verticalement aux autres gestionnaires. Elle met aussi bien en évidence la façon dont le travail est réparti et attire l'attention sur les experts des différents secteurs. Elle conserve l'unité de commandement, principe important d'une saine gestion. De plus, les gestionnaires bénéficient de services spécialisés pour prendre leurs décisions, tout en laissant au gestionnaire hiérarchique l'autorité nécessaire pour faire son travail, de même que la responsabilité de celui-ci.

Ce modèle a pour inconvénient des possibilités de mésententes entre les personnes de la structure hiérarchique, qui prennent les décisions, et celles relevant de l'état-major, c'est-à-dire 
qui jouent un rôle de conseiller, surtout lorsque la décision se révèle erronée ou a des conséquences non désirées. Une autre source de friction apparaît lorsque les personnes qui ont un rôle de conseiller ou de consultant se mêlent de prendre des décisions. À noter encore que les services d'état-major peuvent être coûteux et que ce système comporte parfois des délais dans la prise de décision. De plus, les conseillers se plaignent parfois du fait qu'ils ne disposent pas d'une autorité suffisante pour introduire des idées innovatrices.

La structure fonctionnelle permet la division du travail entre différents types de spécialistes. Les cadres hiérarchiques n'assument pas de fonctions qui ne relèvent pas de leur spécialisation. Ces fonctions sont attribuées aux responsables qui possèdent les connaissances et les aptitudes particulières. Il s'ensuit que les coûts de formation du personnel sont diminués. De plus, le gestionnaire hiérarchique n'est pas responsable de toutes les tâches et de tout le suivi concernant le service: certaines de ces tâches sont de la responsabilité de professionnels ayant d'autres compétences.

Ce modèle a toutefois l'inconvénient de multiplier le nombre de patrons qui dirigent le même individu et peut engendrer des problèmes de communication et de relations. Il est aussi parfois plus difficile de mettre en place une ligne de conduite. Étant donné que l'autorité fonctionnelle est orientée vers une activité en particulier, il s'ensuit un problème de coordination des tâches. Ainsi, dans un département de service social, le chef de service devra se référer à la direction des ressources humaines dans le cas de problèmes relevant d'une convention collective: cette direction a autorité en matière de relations de travail.

La structure qui préconise le rattachement du personnel à différents programmes semble gagner de la popularité auprès des administrateurs d'hôpitaux, surtout dans les milieux universitaires. Nous en discutons les deux variantes déjà expliquées précédemment, l'une de type matriciel et l'autre de type PatientFocused Care.

La structure matricielle « constitue un compromis entre la départementalisation fonctionnelle et la départementalisation selon le produit ${ }^{10}{ }_{\text {}}$. Cette structure matricielle, selon les variantes retenues, peut se retrouver dans un modèle d'organisation basé sur la notion de programme. Elle permet à un gestionnaire responsable d'un projet ou d'un programme d'intégrer des services spécialisés sans faire de changement dans l'ensemble de 
la structure de l'organisation. Elle est efficace si le projet ou le programme est bien défini par écrit, si les échéances et les mandats sont précis et si les rôles de chaque membre de l'équipe sont très minutieusement décrits. Elle accroît la flexibilité et la souplesse de l'organisation qui peut affecter une personne à différents projets. Elle permet la définition précise des résultats et rattache ces derniers aux personnes qui sont responsables de la réalisation du programme. Enfin, elle maintient le principe de l'évaluation du personnel par un professionnel de même discipline.

Cependant, dans ce type de structure, la personne qui travaille à plus d'un projet a plusieurs patrons, ce qui complique la communication et peut engendrer des conflits interpersonnels. Cette dynamique du jeu de la diversité des personnalités et des zones de pouvoir est aussi visible dans les relations entre gestionnaires fonctionnels et gestionnaires hiérarchiques responsables de programmes. Elle peut engendrer des difficultés et déteindre sur les relations avec les membres de l'équipe.

Le modèle Patient-Focused Care est plus intégrateur et a pour avantage de laisser l'autorité à un seul gestionnaire qui a un pouvoir de décision dans la gestion de son programme. La proximité physique des membres de l'équipe-programme est un atout et permet de diminuer les déplacements, ce qui devrait laisser plus de temps pour les services à la clientèle. Cette même proximité des professionnels favorise la coordination, ce qui diminue le temps de gestion et augmente encore une fois le temps consacré à la clientèle. Enfin, certains prétendent que cette façon de faire offre parfois une occasion d'augmenter la polyvalence des membres de l'équipe qui auraient à jouer différents rôles. Ces conditions de travail devraient engendrer une réduction des coûts de fonctionnement du programme. Comme la structure matricielle, ce mode d'organisation permet l'imputation des coûts aux différentes unités ou aux différents programmes.

Dans un tel modèle, le service social, comme les autres professions, est exposé à perdre de sa représentativité et de son influence au sein de la structure hiérarchique, et les professionnels n'ont plus de lieu de regroupement spécifique. Ils ressentent alors plus d'isolement et doivent eux-mêmes prévoir des endroits et des moments de rencontre entre eux pour continuer à se soutenir mutuellement, à se développer et à se stimuler professionnellement. De plus, le travailleur social ainsi que les professionnels d'autres disciplines affectés à un seul programme voient leurs champs de compétence limités à une seule 
problématique, ce qui peut rétrécir leur vision professionnelle, leur faire perdre de vue l'ensemble des objectifs de l'organisme et la qualité du milieu environnant dans lequel le centre hospitalier est intégré.

Ce style de structure dit Patient-Focused Care conduit à un morcellement de la vision sociale de l'ensemble des besoins de la clientèle de l'organisme, vision importante pour une analyse globale des besoins de cette clientèle. En lui-même, il comporte des risques de compétition inter-programmes et on peut alors observer un cloisonnement du programme sur lui-même et un repliement des professionnels limitant leur horizon à leur unité d'intervention. De plus, les différents professionnels étant appelés parfois à jouer un rôle pour lequel ils ne sont pas formés, ils sentent alors leur manque de compétence et éprouvent un sentiment de gêne vis-à-vis de la clientèle à desservir. Enfin, la souplesse que l'on retrouvait dans la structure matricielle et qui permettait de passer d'une unité à l'autre se perd ici facilement, dès que le volume de clientèle est élevé.

Comme on le voit, aucun mode d'organisation n'est parfait: chacun comporte ses avantages et ses inconvénients. Le point le plus important est de choisir une structure qui convient bien à la conjoncture sociale et économique du moment, qui répond à la lecture des besoins spécifiques de la clientèle et, surtout qui permet d'être à l'affût des nouveaux besoins en émergence. Il ne faut pas oublier que la clinique, la recherche et l'enseignement s'alimentent les uns et les autres, et que la structure organisationnelle doit permettre leur développement réciproque en service social comme ailleurs. De plus, il faut prévoir des phases de transition avant d'en arriver à l'implantation du modèle choisi, ce qui devrait se faire par étapes.

\section{LE SERVICE SOCIAL EN MILIEU HOSPITALIER : LES CONDITIONS DE VIE ET LES DÉFIS}

Ce que nous venons de dire nous amène à préciser la mission des travailleurs sociaux et du service social en milieu hospitalier au Québec en cette fin de siècle:

Faire valoir dans le milieu hospitalier les valeurs du service social et sa philosophie d'intervention et principalement centrer son attention sur les besoins sociaux de la personne, de ses proches et de son environnement. 
Les remarques qui suivent visent à préciser quelques façons de s'assurer de l'efficacité du travail professionnel, du rayonnement du service social et de la compréhension de la mission sociale en milieu hospitalier.

\section{La gestion du service social en milieu hospitalier}

Le gestionnaire du service social en milieu hospitalier doit s'assurer qu'il a un contrat de service. Celui-ci, en plus d'établir les objectifs de travail, doit préciser les modes d'évaluation des services offerts. C'est l'outil qui permet aux travailleuses et aux travailleurs sociaux d'être efficaces et qui rend légitimes leur compétence et leur intervention.

En ce qui regarde la gestion du service social et de son personnel, il faut distinguer la responsabilité professionnelle et la gestion du programme. La responsabilité professionnelle demeure celle des travailleurs sociaux et ne peut être déléguée à aucun autre type de professionnel. À titre d'exemple, l'Ordre professionnel des travailleurs sociaux assure au public et aux usagers un contrôle de la pratique professionnelle de ses membres et a, à son service, un comité de discipline. Il demeure donc de la responsabilité des professionnels du service social de fixer des objectifs sociaux en lien avec les objectifs poursuivis par le programme institutionnel ou par le milieu hospitalier, d'établir le diagnostic social et de choisir les moyens d'intervention appropriés. Si l'employeur veut contester une intervention, il devra le faire auprès de pairs aptes à juger de la pratique professionnelle ou auprès d'un organisme habilité à le faire. En ce qui a trait à la gestion du programme, la personne en autorité peut être travailleur social ou professionnel d'une autre discipline. Son rôle vise l'atteinte des objectifs du programme, la bonne marche des activités et la supervision technique du personnel.

Sur le plan de la structure d'organisation rien n'empêche les responsables du service social ou les intervenants de s'unir pour proposer une structure qui permettrait de rattacher les intervenants au programme en ce qui a trait à la gestion des activités et à l'atteinte des objectifs du programme, et à un de leurs pairs pour ce qui regarde les actes professionnels. Une telle façon de faire favoriserait le développement d'un personnel plus efficace et plus adéquat et assurerait un contrôle de la pratique professionnelle intégrée à l'organisation. Elle assurerait aussi une vision sociale de l'ensemble de la clientèle et ferait en sorte que 
cette vision se reflète à tous les niveaux hiérarchiques de l'organisation. Le succès d'une telle opération dépend en partie de la crédibilité de ceux qui en font la démarche, de leur pouvoir de conviction et de leur ténacité. Dans cette ligne de pensée, il ne faut jamais perdre de vue les objectifs poursuivis par le centre hospitalier et toujours utiliser des arguments qui relient à ces objectifs les propositions faites, en mettant bien en évidence cette relation.

Comme autres moyens d'augmenter la visibilité et la crédibilité de l'intervention du service social, soulignons, entre autres, les points suivants.

- Être présent à tous les niveaux de l'organigramme en offrant ses services ou en se faisant élire aux postes d'influence.

- Saisir toutes les occasions de prendre sa place et de jouer un rôle dans les centres de décision, même si ces derniers ne sont pas, en apparence, directement reliés à sa compétence professionnelle ou à sa fonction spécifique dans l'établissement.

- Faire valoir ses compétences professionnelles et offrir ses services partout où l'on traite de clientèle, de services à la communauté et d'autres aspects sociaux, comme :

- l'orientation-patient dans la gestion par programme-clientèle ;

- la gestion de la qualité et des relations avec la communauté ;

- l'élaboration d'outils de suivi de la qualité, principalement en ce qui a trait à la satisfaction de la clientèle et à la gestion des plaintes.

- Permettre au centre hospitalier de jouer un rôle de premier plan dans le maillage avec les partenaires (établissements, organismes communautaires, etc.) à l'intérieur de la Direction de la qualité et des relations avec la communauté.

et

- Centrer son action sur le patient et ses proches et les mettre en évidence à chaque occasion.

Dans le contexte compétitif du milieu hospitalier, acquérir les notions de gestion pour pouvoir parler le même langage que les gestionnaires est un atout important. Une formation complémentaire dans cette discipline peut se révéler un moyen utile à 
la promotion du rôle du service social. Il faut se faire un devoir, comme groupe de professionnels, de toujours accepter l'offre qu'on nous fait de participer à la gestion de l'établissement, à quelque niveau que ce soit. C'est une question de priorité de travail et une bonne façon de faire valoir sa compétence et d'établir un réseau d'appui et des alliances dans l'organisation.

\section{La recherche: une condition de pratique et une nécessité en milieu hospitalier universitaire}

Le milieu universitaire est un milieu où la recherche s'intègre à toutes les activités, et les centres hospitaliers universitaires (CHU) en particulier n'y échappent pas. C'est une habitude pour les professionnels de toutes les disciplines d'intégrer une démarche de recherche à la pratique clinique. Les travailleurs sociaux ne sauraient échapper à cette règle. En plus de l'évaluation de l'efficacité des programmes pour répondre aux besoins de la clientèle, les travailleurs sociaux doivent acquérir des habiletés de recherche permettant de prendre leur place dans des projets de pointe. L'association avec les milieux universitaires de recherche en service social s'impose comme stratégie d'affirmation de la compétence professionnelle. Cette dernière passe par la recherche constante de modèles de pratique de pointe. Ce souci de recherche nécessite, dans bien des cas, une transformation de mentalité et de culture professionnelle. Les exigences de qualifications des intervenants doivent évoluer vers la nécessité d'avoir un doctorat comme base de recrutement de travailleurs sociaux pour certains postes.

\section{L'enseignement : un apport important à développer}

De longue date, les travailleurs sociaux en milieu hospitalier ont participé à l'enseignement par l'offre de stage. Cette contribution est essentielle à l'acquisition des compétences spécifiques des futurs travailleurs sociaux en milieu hospitalier. La contribution à l'enseignement passe souvent par la collaboration des travailleurs sociaux du milieu hospitalier avec les professeurs à la formation des étudiants. Cette association clinicien-professeur est une formule appréciée qui met en évidence la complémentarité des expériences et des connaissances et renforce le lien entre la théorie et la pratique. La participation à l'enseignement peut aussi - et souvent doit - prendre la forme d'enseignement 
aux collègues d'autres professions qui travaillent dans le milieu. Elle s'exprime encore par la participation aux différents colloques soit à l'intérieur du centre hospitalier, soit à l'extérieur.

Somme toute, les travailleurs sociaux en milieu hospitalier acquièrent des compétences spécifiques dans le réseau de la santé. Il est essentiel qu'ils partagent ces connaissances, surtout dans un contexte d'alliance formelle entre le centre hospitalier universitaire (CHU), les centres hospitaliers affiliés (CHA) et les instituts.

\section{Le défi technologique}

Le service social n'échappe pas à la tendance du défi technologique. Le service social en milieu hospitalier y est encore beaucoup plus exposé. Les applications technologiques, surtout les applications informatiques en service social, se sont souvent développées en premier lieu dans les services sociaux hospitaliers. La recherche de pointe repose sur la maîtrise et l'utilisation de ces technologies, même en service social. L'informatisation constitue un moyen privilégié pour aider à structurer l'intervention, à la conceptualiser, à la systématiser et à l'évaluer. Le meilleur moyen de contrôler l'usage et l'évolution de la technologie est de s'en approprier la conception.

Là encore, un changement de mentalité et de culture s'impose à notre profession pour qu'elle joue un rôle proactif, sans quoi les autres types d'intervenants sauront s'approprier nos secteurs de compétence en élaborant des outils modernes auxquels nous ne pourrons que réagir, étant réduits au statut de simples utilisateurs ou exécutants.

\section{EN GUISE DE CONCLUSION}

Le milieu hospitalier est de plus en plus influencé par des modèles administratifs qui viennent s'ajouter aux modèles cliniques médicaux traditionnels. Les professionnels ont de la difficulté à s'y retrouver, car ces modèles imposent une rupture avec la tradition. Le service social et ses objectifs sociaux se greffent en troisième lieu dans un contexte où l'aspect social n'est pas la raison d'être de l'établissement.

Cependant, le service social en milieu hospitalier propose plusieurs défis stimulants qui sont susceptibles d'attirer des 
personnes très compétentes. La compétence professionnelle s'exerce à l'intérieur d'une structure administrative sur laquelle les travailleurs sociaux doivent exercer une influence, non seulement au moment de son implantation, mais tout au cours de son application et de ses mutations, et ce, en conformité avec la mission du service social:

Faire valoir dans le milieu hospitalier les valeurs du service social, sa philosophie d'intervention et principalement centrer son attention sur les besoins sociaux de la personne, de ses proches et de son environnement.

La connaissance du modèle administratif devient un atout important pour exercer sa fonction de travailleur social, surtout comme responsable d'un groupe de professionnels. De plus, la compétence clinique en milieu hospitalier et la reconnaissance de l'apport spécifique de notre profession passent par la contribution à l'enseignement et à la recherche ainsi que par le défi technologique.

Il est parfois difficile ou de mauvaise tactique de s'opposer à un mode d'organisation, mais il est très souvent possible d'exprimer et de vendre son point de vue pour l'améliorer, d'établir des conditions nécessaires au maintien et au développement de l'expertise spécifique des différents professionnels, de tirer profit des avantages et de minimiser les inconvénients. À notre avis, le modèle d'organisation basé sur la notion de programme, dans sa variante de type matriciel, nous semble proposer les meilleures conditions de travail dans la conjoncture actuelle. Il permet d'offrir les avantages du regroupement professionnel de la structure fonctionnelle, offre une souplesse d'ajustement en fonction des besoins de la clientèle de l'ensemble de l'organisme, permet une vision globale et évolutive de la réalité sociale de la clientèle, assure l'intégration des travailleurs sociaux à une équipeprogramme et centre l'attention de cette équipe sur la clientèle. Dans ce mode d'organisation, le chef du service social devient le porte-étendard de la mission sociale auprès des différents niveaux hiérarchiques.

Enfin, quel que soit le modèle administratif, il revient aux travailleurs sociaux de mettre à profit leur analyse critique sociale, de participer activement en particulier à la triple mission du centre hospitalier universitaire : soins et services, enseignement, recherche. La personne, ses proches, son environnement ont été et sont toujours, selon les valeurs du service social, des partenaires de premier plan dans la recherche du bien-être et de la santé. 
Il s'agit en somme d'orienter notre action vers une démarche $\mathrm{d}^{\prime}$ " empowerment»; si cette dernière est bonne pour les clients, elle vaut aussi pour les travailleurs sociaux dans un contexte de travail! C'est un choix de priorités qu'il faut sans cesse exercer.

\section{Notes}

1. Plusieurs des notions théoriques expliquées et des exemples apportés dans ce texte ont été inspirés par la présentation du 20 septembre 1996 à la Journée d'échanges professionnels de l'Association des praticiens de service social en milieu de santé au Québec. Nous remercions bien sincèrement les personnesressources qui ont participé à ces échanges, en particulier Marie Breton, alors travailleuse sociale au Centre hospitalier universitaire de Québec, et Martin Poulin, professeur titulaire à l'École de service social.

2. La Charity Organization Society (C.O.S.) de Londres est un organisme offrant une aide à caractère philanthropique.

3. Le lecteur pourrait trouver un peu arides certains des concepts administratifs que l'on rencontre dans les pages qui suivent, concepts qui sont expliqués brièvement dans les notes. Pour des renseignements supplémentaires, on pourra se référer aux ouvrages cités dans ces notes.

4. Selon Pierre-G. Bergeron, La gestion moderne - Théorie et cas, Boucherville, Gaëtan Morin, 2 éd., 1989, p. 199.

La structure hiérarchique «est formée de personnes responsables de la réalisation des objectifs principaux de l'entreprise (cadres hiérarchiques) et d'individus qui participent à la réalisation des grands objectifs de l'entreprise en aidant, en assistant et en consultant les cadres hiérarchiques dans les domaines techniques ou spécialisés (cadres de l'“ État Major") ». Les travailleurs sociaux du département et le chef du service social sont des personnes-ressources dans l'hôpital. Le chef de service social offre aussi un service spécialisé dans son domaine aux cadres hiérarchiques de l'hôpital.

5. Par procédé, on entend ici le processus par lequel le service est offert à la clientèle.

6. Selon Pierre-G. Bergeron. op. cit., p. 199.

La structure fonctionnelle est celle «où des spécialistes dans un domaine particulier peuvent donner des ordres [administratifs] à des employés qui ne font pas partie de leur propre structure, mais qui occupent plutôt des postes dans une autre unité organisationnelle sous la responsabilité d'un cadre hiérarchique ».

7. Idem, p. 213.

«Dans le but de réaliser le mieux possible ses objectifs, l'organisation doit être structurée en sections, et ses principales activités doivent être regroupées selon un ordre logique; ce processus est celui de la départementalisation. [...] Pour être efficace, une entreprise doit combiner certains de ces modes de départementalisation. »

La départementalisation en fonction des canaux de distribution (ex. : le centre hospitalier, l'école) structure l'organisation en fonction de ce critère.

8. Dans Pierre-G. Bergeron, op. cit., p. 196.

«La structure matricielle est étroitement liée à la gestion de projet. [...] La principale différence entre l'organisation de projet et l'organisation matricielle 
est qu'au sein de cette dernière le personnel et le matériel des unités fonctionnelles sont prêtés à une unité quelconque pour réaliser un programme. »

9. Dans Raymond Beaudry et Bruno Fabi, «La structure matricielle: une solution possible mais exigeante», Administration hospitalière et sociale, 1983, p. 37-42.

«Les ressources (professionnelles) sont en permanence rattachées à leur fonction ou département, mais elles sont assignées à des projets ou programmes selon les besoins » (p. 38).

10. Dans Pierre-G. Bergeron, op. cit. Voir note 8.

\section{Références bibliographiques}

ASSOCIATION DES HÔPITAUX DU QUÉBEC (AHQ) (1994). Regard sur les tendances en matière d'organisation des établissements de santé, $67 \mathrm{p}$.

BARTletT, Harriett M. (1961). Social Work Practice in Health Field, National Association of Social Workers, p. 20.

BEAUDRY, Raymond, et Bruno FABI (1983). "La structure matricielle: une solution possible mais exigeante», Administration hospitalière et sociale, p. 37-42.

Bergeron, Pierre-G. (1989). La gestion moderne - Théorie et cas, Boucherville: Gaëtan Morin, 565 p.

COLlÈge CANADIEN DES DIRECTEURS DES SERVICES DE SANTÉ (1994). Program Management and Beyond: Management Innovations in Ontario Hospitals, Ottawa: P. Leatt, L. Charles et C. Airds, 89 p.

ÉMOND, Marie (1976). "Comment on transforme les travailleurs sociaux en bureaucrates », p. 71-76, dans M. Émond, J. Lindsay et C. Perreault (1985), Le travail social et la santé au Québec, Behaviora, $480 \mathrm{p}$.

KoOnTZ, H., et C. O’Donnel (1980). Management - principes et méthodes de gestion, Montréal: McGraw-Hill, 618 p.

MACMAN, Martin (1990). "Social Work in Health Setting Historical Review», p. 7-22, dans K.W. Davidson et Sylvia S. Clarke, Social Work in Health Care: a Handbook for Practice, New York-London: The Haworth Press, 935 p.

NinACS, William A. (1995). «Empowerment et service social : approches et enjeux", Service social, vol. 44, n 1, p. 69-93.

PERron Jules (1986). Administration et services sociaux, Chicoutimi : Gaëtan Morin, 285 p.

SABETTA, Y. (1981). «Le service social hospitalier dans les années 1980 », Service social, vol. $30, \mathrm{n}^{\text {os }} 1$ et 2, p. 7-18.

ST-ARNAUD, Georgette (1967). "Rôle du travailleur social en milieu médical ", Service social, vol. 16, n ${ }^{\text {os }} 1$ et 2, p. 3-29.

TAYLOR ROSSINGER, Elizabeth (1985). «Tableau synoptique sur le développement du social en milieu de santé », dans M. Émond, J. Lindsay et C. Perreault, Le travail social et la santé au Québec, Behaviora, p. 437-470. 
